\title{
Propiedades psicométricas escala satisfacción y frustración necesidades psicológicas (ESFNPB) en universitarios chilenos
}

\section{Psychometric Properties of the Psychological Needs Satisfaction Frustration Scale (BPNSFS) in Chilean University Students}

Milenko Del Valle*1 iD Universidad de Antofagasta, Antofagasta, Chile. ORCID: https://orcid.org/0000-0003-3739-1001

Lennia Matos 2 (D) Pontificia Universidad Católica del Perú, Lima, Perú. ORCID: https://orcid.org/0000-0003-2271-4816

Alejandro Díaz ${ }^{3}$ Universidad de Concepción, Concepción, Chile. ORCID: http://orcid.org/0000-0002-3090-5463

María Victoria Pérez ${ }^{4}$ Universidad de Concepción, Concepción, Chile. ORCID: https://orcid.org/0000-0002-3697-7978

Jorge Vergara ${ }^{5}$ (D) Universidad de Concepción, Concepción, Chile. ORCID: https://orcid.org/0000-0003-3655-813X

${ }^{1}$ Dr. (c) en Psicología. Profesor asociado de la Facultad de Ciencias Sociales, Artes y Humanidades.

${ }^{2}$ Dra.en Psicología. Profesora asociada de la Facultad de Psicología. ${ }^{3}$ Dr. en Psicología. Profesor Titular y Director del Doctorado en Psicología de la Facultad de Ciencias Sociales. ${ }^{4}$ Dra. en Psicología. Profesora Titular de la Facultad de Ciencias Sociales. ${ }^{5}$ Dr. (c) en Psicología. Programa de Doctorado en Psicología.

Recibido 14-11-17 Revisado 20-10-17 Aprobado 24-01-18 En línea 25-01-18

\section{*Correspondencia}

Email: mdelvalle@uantof.cl

\section{Citar como:}

Del Valle, M., Matos, L., Diaz, A., Pérez, M.V. \& Vergara J. (2018). Propiedades psicométricas escala satisfacción y frustración necesidades psicológicas (ESFNPB) en universitarios chilenos. Propósitos y Representaciones, 6(1), 301-350. doi: http://dx.doi. org/10.20511/pyr2018.v6n1.202

(c) Universidad San Ignacio de Loyola, Vicerrectorado de Investigación, 2018

(cc) BY-NC-ND Este artículo se distribuye bajo licencia CC BY-NC-ND 4.0 Internacional (http://creativecommons.org/licenses/by-nc-nd/4.0/). 


\section{Resumen}

La presente investigación tiene como propósito analizar las propiedades psicométricas de la Escala de Satisfacción y Frustración de las Necesidades Psicológicas Básicas de Autonomía, Competencia y Relación (ESFNPB) identificadas por la teoría de la autodeterminación (Deci \& Ryan, 2000b), en una muestra de 297 estudiantes universitarios de distintas facultades y carreras pertenecientes a una universidad Chilena. Para lograr el objetivo, mediante un estudio psicométrico por procedimientos confirmatorios, se realizó un análisis factorial confirmatorio (AFC), analizando la propuesta de seis factores realizada por Chen et al. (2015) y se evaluó la consistencia interna de la escala mediante el Alfa ordinal. Los resultados obtenidos con la muestra de estudiantes universitarios indican una buena consistencia interna, alfa $=0.90$ y 0.86 para satisfacción y frustración de las necesidades psicológicas, así mismo los resultados del análisis factorial confirmatorio mostraron un ajuste adecuado del modelo a los datos $\left(\chi^{2} / g l=1.75\right.$; CFI $=0.92 ; \mathrm{IFI}=0.92$; TLI $=0.90 ;$ RMSEA $=.05$ y SRMR $=.05$ ), brindando evidencias de validez de la estructura de seis factores. Según lo anterior se considera que la escala para medir satisfacción y frustración de las tres necesidades psicológicas básicas puede ser utilizada preliminarmente en estudiantes universitarios en el contexto de la educación superior chilena, permitiendo también la relación con otras variables de interés para generar modelos explicativos que permitan profundizar la comprensión de aspectos que son de interés institucional.

Palabras clave: Propiedades psicométricas, Escala ESFNPB, estudiantes universitarios

\section{Summary}

This research work aims to analyze the psychometric properties of the Basic Psychological Needs Satisfaction and Frustration Scale (BPNSFS) -autonomy, competence and relatedness- identified by the self-determination 
theory (Deci \& Ryan, 2000b), in a sample of 297 university students from different faculties and programs belonging to a Chilean university. To achieve the objective, through a psychometric study by confirmatory procedures, a confirmatory factor analysis (CFA) was performed, analyzing the six-factor proposal developed by Chen et al. (2015), and the internal consistency of the scale was evaluated using the ordinal Alpha. The outcomes obtained from the sample of university students indicate a good internal consistency, Alpha $=0.90$ and 0.86 for psychological needs satisfaction and frustration. Besides, the outcomes of the confirmatory factor analysis showed an adequate fit of the model $\left(\chi^{2} / g l=1.75 ; \mathrm{CFI}=0.92 ; \mathrm{IFI}=0.92 ; \mathrm{TLI}=0.90 ; \mathrm{RMSEA}=.05\right.$ and $\mathrm{SRMR}=.05)$ to the data, showing evidences of the validity of the six-factor structure proposed. According to the foregoing, it is considered that the scale to measure satisfaction and frustration of the three basic psychological needs can be used initially in university students in the higher education of Chile, thus, allowing the relationship with other variables of interest to generate explanatory models that allow going in depth the understanding of aspects that are of institutional interest.

Keywords: Psychometric properties, BPNSFS Scale, university students. 


\section{Introducción}

La transición de la educación secundaria a la universitaria está determinada por una serie de condicionantes que interactúan y que influyen en el aprendizaje, el desempeño académico y en la permanencia o abandono de los estudios universitarios, está relacionada con la calidad del proceso educativo y con evidentes repercusiones en aspectos psicosociales y socioeconómicos en los estudiantes (Fernández-Hileman, Corengia \& Durand, 2014; Garbanzo, 2012).

El abandono en la educación superior es un tema de preocupación para las instituciones, ya sea por las presiones sociales, de las familias, de las fuentes de financiamiento y por el impacto que genera en el debilitamiento del capital humano avanzado de los países. En el caso de Chile este fenómeno ha despertado un interés generalizado, ya que se considera en promedio que por lo menos la mitad de los estudiantes que ingresan a la educación superior abandonan los estudios antes de completar su formación profesional y obtener un título o grado académico (Abello et al., 2012; Donoso \& Schiefelbein, 2007).

Los aportes teóricos y empíricos, para determinar las causas que inciden en el desempeño académico y en la permanencia o abandono en la educación superior, han aumentado en el último periodo, considerando factores de orden personal, social (Barahona, 2014), así como determinantes educacionales, como el caso de los hábitos de estudios (Chilca, 2017), socioculturales y económicos (Catalán \& Santelices, 2014; Garcés \& Arriagada, 2015). Los estudios de los factores que intervienen en el proceso de aprendizaje destacan la importancia que adquieren las variables cognitivo-motivacionales como mediadoras del éxito o fracaso académico (Miñano \& Castejón, 2011).

La literatura especializada ha mostrado abundante evidencia de la importancia que tiene la motivación académica en el proceso de aprendizaje de los estudiantes y alta relación con el aprendizaje autorregulado y el autoconcepto académico (Carranza \& Apaza, 2015). En general, se asume 
que la motivación hace referencia a aquellos factores internos que energizan, impulsan y dirigen al estudiante a realizar una serie de actividades propuestas en el proceso de aprendizaje, actuando como mediadora en la adquisición de los contenidos curriculares, persistencia, finalidad del comportamiento y el desempeño académico (Stover, Uriel, De la Iglesia, Freiber \& Liporace, 2014; Valenzuela, Muñoz, Silva-Peña, Gómez \& Precht, 2015).

Una de las teorías motivacionales actuales es la teoría de la autodeterminación (TAD; Deci \& Ryan, 2000b), que logra superar la orientación exclusiva hacia la meta o logro académico, asumiendo la motivación como el resultado de las tendencias internas de la persona que la impulsa a realizar conductas en ausencia de recompensas externas, se sustenta en la idea del ser humano como agente causal y orientado para el crecimiento, con una tendencia natural para la integración de sus elementos psíquicos y para su integración en estructuras sociales cada vez más amplias y complejas (Ryan \& Deci, 2000b).

Esta teoría reconoce que la motivación tiene una naturaleza multidimensional con distintos niveles situados en un continuo cumpliendouna función descriptiva, que va desde los niveles más altos de autodeterminación y por lo tanto con una elevada motivación intrínseca, hasta el nivel más bajo de motivación, denominada desmotivación, encontrándose entre ellas distintos tipos de motivación extrínseca, según el nivel de autodeterminación que presenten las personas; externa, introyectada, identificada e integrada, por lo que en la medida que la motivación regulada externamente se oriente hacia la regulación integrada, los comportamientos se vuelven menos dependiente de controles externos y por lo tanto más autodeterminados (Faye \& Sharpe, 2008; Moreno, Silveira \& Alias, 2015).

La TAD propone la existencia de tres necesidades psicológicas básicas; necesidad de autonomía, competencia y relación que influyen en la motivación intrínseca de los individuos y que los hacen actuar de una determinada manera (Doménech \& Gómez, 2011; Ryan \& Deci, 2000a). La autonomía se refiere a que la persona siente que es la causante de su conducta, no significa 
independencia más bien aceptación interna y compromiso con la conducta motivada. La competencia se da cuando la persona se siente eficaz con su conducta, logra interactuar efectivamente con el entorno experimentando la oportunidad para expresar o desarrollar sus capacidades. Finalmente la necesidad de relación se produce cuando una persona se siente vinculada $o$ comprendida por los demás, están relacionados con autenticidad con otros significativos y experimentan un sentido de pertenencia, necesidades que han sido identificadas como básicas en el desarrollo psicológico óptimo y el bienestar general (Deci \& Ryan, 2000b; Tian, Chen \& Huebner, 2014).

Estas tres necesidades psicológicas, consideradas en términos organísmicos y funcionales, son innatas y nutrientes esenciales para el crecimiento psicológico, la integración y el bienestar, desempeñando un papel fundamental en la salud psicológica de los individuos y su satisfacción se asocia con un nivel de funcionamiento más eficaz (Deci \& Ryan, 2000b). Se ha reconocido que ciertos factores ambientales influyen y afectan la satisfacción de las necesidades produciendo comportamientos autodeterminados o bien obstaculizando su satisfacción generando comportamientos no autodeterminados (Faye \& Sharpe, 2008).

Para medir estas necesidades psicológicas, Deci y Ryan (2000a) propusieron el instrumento denominado Basic Psychological Needs Scale (BPNS), que evalúa la satisfacción de estas necesidades tanto a nivel general como en ámbitos específicos, extendiendo su adaptación y utilización en diferentes países y contextos. Posteriormente, se han desarrollado escalas para medir la satisfacción de estas necesidades en distintos ambientes y contextos culturales; en el ejercicio físico, deportivo, en clases de educación física y en educación superior (León, Domínguez, Núñez, Pérez \& MartínAlbo, 2011; Vlachopoulos \& Michailidou, 2006; Wilson, Rogers, Rodgers \& Wild, 2006).

Recientemente, Chen et al. (2015) elaboraron en inglés el instrumento denominado Basic Psychological Need Satisfaction and Frustration Scale (BPNSFS), validado en cuatro culturas e idiomas diferentes: China, Bélgica, 
Estados Unidos y Perú. Los autores del estudio original han entregado evidencias que la escala compuesta por 24 ítems presenta una estructura multidimensional de seis factores, que miden satisfacción y frustración respectivamente de cada una de las necesidades psicológicas. Para realiza el análisis trabajaron con una muestra de 1,051 estudiantes universitarios con una media de edad de 20 años. Los resultados obtenidos en la validación cruzada reconocieron cuatro ítems por necesidad, con una consistencia interna por cada dimensión que están entre 0.64 y 0.89. El modelo de seis factores propuesto por los autores arrojó un buen ajuste, SBS- $\chi^{2}(231)=$ 441.99, $\mathrm{CFI}=0.95, \mathrm{RMSEA}=.04$ y SRMR $=.04$. (Chen et al., 2015).

Posteriormente Cordeiro, Paixão, Lens, Lacante y Luyckx (2016), realizaron la traducción de la BPNSFS al portugués, examinando la validación de constructo con estudiantes portugueses de pregrado. Para evaluar las propiedades psicométricas de la escala utilizaron una muestra de 417 estudiantes universitarios de distintas carreras y con una media de edad de 20 años. Los resultados obtenidos mostraron una buena consistencia interna de la escala que están entre .70 para frustración a la autonomía y de 0.85 para satisfacción de competencia, así mismo el análisis factorial confirmatorio realizado demostró buenos índices de ajuste, $\chi 2(237)=519.128, \mathrm{CFI}=0.95$, RMSEA $=.05$ y SRMR $=.05$. Resultados que apoyan los datos al modelo original de 6 factores (Cordeiro et al., 2016).

En nuestro contexto existe un interés generalizado por el estudio de la motivación académica y de las necesidades psicológicas de los estudiantes universitarios, no obstante y según la revisión realizada sólo se encontraron antecedentes de la validación cruzada de la escala realizada por Chen et al. (2015), que incluyó estudiantes universitarios limeños obteniendo para este grupo valores de consistencia interna adecuados para las seis subescalas; $\alpha=0.74$ (satisfacción autonomía), un $\alpha=0.75$ (satisfacción relación) y un $\alpha=0.78$ (satisfacción competencia), mientras que se obtuvo un $\alpha=0.77$ (frustración autonomía), un $\alpha=0.64$ (frustración relación) y un $\alpha=.67$ 
(frustración competencia). Según lo anterior resulta importante estudiar el funcionamiento de la escala en el contexto de la educación superior chilena.

Considerando lo expuesto el presente estudio tiene como objetivo analizar las propiedades psicométricas, mediante análisis clásico de los ítems, análisis factorial confirmatorio y la consistencia interna de la Escala de Satisfacción y Frustración de las Necesidades Psicológicas Básicas (ESFNPB), en una muestra de estudiantes universitarios chilenos de distintas facultades y carreras, aportando evidencias de su estructura multidimensional para su uso en la educación superior.

\section{Método}

\section{Diseño y Participantes.}

Se utilizó un estudio de tipo cuantitativo con un diseño instrumental para evaluar las propiedades psicométricas de la escala que mide satisfacción y frustración de las necesidades psicológicas básicas (Ato, López \& Benavente, 2013). El tipo de muestreo utilizado fue no probabilístico incidental.

Para la realización del estudio se trabajó con un muestra compuesta por 297 estudiantes universitarios de primer y segundo año de la Universidad de Antofagasta, con edades comprendidas entre los 18 y 40 años $(M=20.6$, $\mathrm{DE}=2.29$ ), de ellos 194 eran mujeres y 103 hombre, que representan el $65.3 \%$ y $34.7 \%$ respectivamente. Los estudiantes que participaron de manera voluntaria en el estudio pertenecen a cuatro diferentes facultades: Ciencias Sociales, Artes y Humanidades, Educación, Ingeniería y Facultad de Medicina y Odontología.

\section{Instrumento.}

El instrumento original, Basic Psychological Need Satisfaction and Frustration Scale (BPNSFS) se validó en cuatro culturas e idiomas diferentes 
(Chen et al., 2015), la versión en español de la escala fue adaptada y validada con estudiantes universitarios peruanos, específicamente de Lima, utilizando la traducción lingüística realizada por expertos en el idioma y la validez de contenido según juicio de expertos en la teoría de la autodeterminación.

La escala está compuesta por 24 ítems agrupados en seis factores que miden satisfacción y frustración de cada una de las necesidades psicológicas básicas, propuestas por la teoría de la autodeterminación; satisfacción/ frustración de la necesidad de autonomía, competencia y relación. Para la dimensión autonomía, 8 ítems que miden sentimientos en la posibilidad de elegir libremente y comprometerse con la elección (p. ej.: "Siento que tengo la libertad y posibilidad de elegir las cosas que asumo"). Dimensión competencia con 8 ítems que evalúa la capacidad en la realización de actividades y alcanzar metas con éxito (p. ej.: "Siento que puedo hacer las cosas bien"). Y la dimensión relación compuesta por 8 ítems que mide la relación con otros y el sentido de pertenencia (p. ej.: "Siento que les importo a las personas que me importan"). Para cada caso, 4 ítems miden satisfacción y 4 ítems miden frustración de las necesidades. Los 24 ítems son evaluados a través de una escala de respuesta con formato tipo Likert de 5 puntos que van desde 1 (totalmente falso) a 5 (totalmente verdadero).

Específicamente la versión en español que se utilizó en el presente estudio fue autorizada, para su aplicación con estudiantes universitarios chilenos, por la investigadora peruana quien formó parte del equipo de investigación que realizó esta versión de la prueba recogiendo evidencias de validez y confiabilidad en cuatro muestras de países distintos (Chen et al., 2015).

\section{Procedimiento.}

La versión de la escala en español fue administrada de forma piloto a un grupo de ocho estudiantes universitarios de distintos niveles para verificar la compresión de cada uno de los ítems, los resultados de esta aplicación piloto no presentaron ningún problema de comprensión. 
La administración final de la escala se realizó en dependencias de la Universidad de Antofagasta, Chile. En primer lugary antes de la aplicación del instrumento se contactó con Jefes de Carrera y /o Directores de Departamento para solicitar su colaboración y explicar los objetivos de la investigación, una vez obtenida su autorización se coordinó día y horario para la aplicación del instrumento. Se solicitó la participación voluntaria y anónima de estudiantes entregándoles carta de consentimiento informado, documento que explicaba los objetivos del estudio, el tiempo de duración, indicaciones para responder el instrumento y el resguardo de la confidencialidad tanto de los datos como de los resultados obtenidos. Dicha aplicación se realizó de forma colectiva en la misma sala de clases a fines del segundo semestre del periodo académico 2016. Los aspectos éticos de la investigación se fundamentan sobre la base de tres premisas básicas: el respeto a las personas (principio de autonomía), la búsqueda del bien (principios de beneficencia y no maleficencia) y el principio de justicia. La manera de hacer operativo estos principios, declarados universales en el campo de la investigación científica, se realizó por medio del consentimiento informado y firmado, determinando los riesgos y beneficios para los participantes en el estudio (Agar, 2004; Mondragón, 2007).

\section{Análisis de datos.}

Con el objetivo de comprobar la estructura factorial de la escala en una muestra de estudiantes universitarios chilenos, se recogieron evidencias de validez factorial mediante la utilización de paquete estadístico SPSS V.23 y el programa AMOS V. 23. Para el análisis de la validez factorial del instrumento se utilizó un análisis factorial confirmatorio (AFC), a través del método de máxima verosimilitud, según el modelo y la estructura original de la escala. El análisis de la normalidad de los datos se trabajó examinando los valores univariados de asimetría y curtosis. El AFC realizado permitió analizar y comprobar si el modelo teórico propuesto se ajusta a los datos utilizados (Roth, 2012) para apoyar la validez del modelo propuesto de 
seis factores que miden satisfacción y frustración de las tres necesidades psicológicas básicas de autonomía, competencia y relación realizada por Chen et al. (2015). El ajuste del modelo se comprobó mediante diferentes índices de bondad de ajuste (Boomsma, 2000), con el objetivo de responder a los propósitos y análisis de la investigación. Se utilizaron como índices de buen ajuste cuando el ratio entre el valor de chi-cuadrado y los grado de libertad era menor a 3, $\chi^{2} /$ grados de libertad $\left(\chi^{2} / g l\right)$ (Kline, 2005), índice de bondad de ajuste comparativo (CFI), cuyo valor debe ser igual o superior a 0.90, índice de ajuste incremental (IFI) que indica mejoras en el ajuste del modelo por grados de libertad y que debe ser igual o superior a 0.90 , índice de Tucker-Lewis (TLI) que debe ser igual o superior a 0.90 (Hair, Black, Babin, Anderson \& Tatham, 2006; Shumacker \& Lomax, 1996). Se incluyó además como índice de buen ajuste la raíz del residuo cuadrático promedio de aproximación (RMSEA) que debe ser menor a .06 o asume un valor máximo de .08 y la raíz del residuo cuadrático promedio (SRMR) que debe ser igual o inferior a .08 (Byrne, 2009; Hu \& Bentler, 1999). La consistencia interna se evaluó mediante el alfa ordinal, que ha demostrado mayor nivel de precisión para escalas de respuestas ordinales y dicotómicas (Elosua \& Zumbo, 2008), mediante el programa Microsoft Office Excel.

\section{Resultados}

\section{Análisis descriptivos preliminares.}

Para la realización de los análisis descriptivos se procedió a calcular la asimetría y curtosis, bajo el supuesto de una distribución normal de los datos. Como se muestra en la tabla 1 todos los valores de asimetría univariada se encuentran igual o por debajo del valor 2, oscilando entre .097 (ítem 2) y -2.03 (ítem 17), mientras que los valores de la curtosis, que debe ser igual o inferior al valor 7, oscilaron entre -.050 (ítem 13) y 5.11 (ítem 17), cumpliendo así con el criterio de normalidad univariada (Curran, West y Finch, 1996). Así 
mismo la escala de satisfacción y frustración de las necesidades psicológicas básicas (ESFNPB) presenta medias entre 4.59 (ítem 17) y 1.59 (ítem 4), desviaciones estándar entre 1.30 (ítem 24) y .740 (ítem 17).

\section{Tabla 1.}

Estadísticos descriptivos de los datos en la muestra de estudiantes universitarios.

\begin{tabular}{|c|c|c|c|c|c|}
\hline & Ítem & $M$ & $S D$ & Asimetría & Curtosis \\
\hline 1 & Siento que tengo la libertad y la posibilidad de elegir las cosas que asumo. & 4.16 & .976 & -1.05 & .654 \\
\hline 2 & $(*)$ & 3.04 & 1.06 & .097 & -.504 \\
\hline 3 & Siento que les importo a las personas que me importan. & 4.25 & .962 & -1.23 & 1.01 \\
\hline 4 & $(*)$ & 1.59 & 1.06 & 1.86 & 2.58 \\
\hline 5 & Siento que puedo hacer las cosas bien. & 4.43 & .816 & -1.62 & 2.88 \\
\hline 6 & $(*)$ & 2.19 & 1.18 & .680 & -.609 \\
\hline 7 & $(*)$ & 3.98 & .993 & -.626 & -.433 \\
\hline 8 & $(*)$ & 2.03 & 1.14 & 1.03 & .305 \\
\hline 9 & $(*)$ & 4.32 & .932 & -1.41 & 1.67 \\
\hline 10 & $(*)$ & 1.77 & 1.11 & 1.35 & .837 \\
\hline 11 & $(*)$ & 4.40 & .756 & -1.23 & 1.24 \\
\hline 12 & $(*)$ & 2.46 & 1.20 & .613 & -.484 \\
\hline 13 & $(*)$ & 4.08 & .946 & -.772 & -.050 \\
\hline 14 & $(*)$ & 2.61 & 1.29 & .345 & -.996 \\
\hline 15 & $(*)$ & 4.32 & .791 & -.891 & -.063 \\
\hline 16 & $(*)$ & 1.79 & 1.10 & 1.37 & .978 \\
\hline 17 & $(*)$ & 4.59 & .740 & -2.13 & 5.11 \\
\hline 18 & $(*)$ & 2.31 & 1.27 & .634 & -.747 \\
\hline 19 & (*) & 4.18 & .964 & -1.11 & .781 \\
\hline 20 & $(*)$ & 2.54 & 1.19 & .477 & -.675 \\
\hline 21 & $(*)$ & 4.36 & .871 & -1.51 & 2.30 \\
\hline 22 & $(*)$ & 1.85 & 1.04 & 1.09 & .440 \\
\hline 23 & $(*)$ & 4.14 & .852 & -.876 & .571 \\
\hline 24 & $(*)$ & 2.14 & 1.30 & .932 & -.289 \\
\hline
\end{tabular}

Nota: (*) Se ha omitido el contenido del item para resguardar los derechos de autor, según lo sugerido por los creadores del instrumento original. 
Según los resultados obtenidos en la matriz de cargas factoriales de los ítems de la ESFNPB, se confirma satisfactoriamente las dimensiones de la escala original. Según se muestra en la tabla 2 el total de los ítems explican el $55 \%$ de la varianza total. Los valores de las saturaciones factoriales estandarizadas son adecuados siendo todas significativas, alcanzando valores que oscilan entre .43 (ítem 8 ) y .82 (ítem 19), corroborando la estructura de 6 factores propuesta por Chen et al. (2015). Al analizar la correlación entre los seis factores en la tabla 3 se observa que existe una correlación positiva y significativa entre los factores que miden satisfacción de las necesidades psicológicas de autonomía, relación y competencia, situación similar si se observa la correlación entre los factores que miden frustración de estas tres necesidades psicológicas y como era de esperarse una correlación negativa y significativa entre los factores que miden satisfacción y frustración respectivamente. 


\section{Tabla 2.}

Matriz de cargas factoriales de los items de la escala.

\begin{tabular}{|c|c|c|c|c|c|c|c|}
\hline & \multirow[t]{2}{*}{ Ítems } & \multicolumn{2}{|c|}{ Autonomía } & \multicolumn{2}{|c|}{ Relación } & \multicolumn{2}{|c|}{ Competencia } \\
\hline & & $S A T$ & FRUS & $S A T$ & FRUS & $S A T$ & FRUS \\
\hline 1 & $\begin{array}{l}\text { Siento que tengo la libertad y la } \\
\text { posibilidad de elegir las cosas que } \\
\text { asumo. }\end{array}$ & .61 & & & & & \\
\hline 2 & $(*)$ & .67 & & & & & \\
\hline 3 & $(*)$ & .75 & & & & & \\
\hline 4 & $(*)$ & .46 & & & & & \\
\hline 5 & $(*)$ & & .56 & & & & \\
\hline 6 & $(*)$ & & .71 & & & & \\
\hline 7 & $(*)$ & & .59 & & & & \\
\hline 8 & $(*)$ & & .43 & & & & \\
\hline 9 & $\begin{array}{l}\text { Siento que les importo a las personas } \\
\text { que me importan. }\end{array}$ & & & .49 & & & \\
\hline 10 & $(*)$ & & & .80 & & & \\
\hline 11 & $(*)$ & & & .67 & & & \\
\hline 12 & $(*)$ & & & .62 & & & \\
\hline 13 & $(*)$ & & & & .62 & & \\
\hline 14 & $(*)$ & & & & .64 & & \\
\hline 15 & $(*)$ & & & & .65 & & \\
\hline 16 & $(*)$ & & & & .49 & & \\
\hline 17 & Siento que puedo hacer las cosas bien. & & & & & .63 & \\
\hline 18 & $(*)$ & & & & & .73 & \\
\hline 19 & $(*)$ & & & & & .82 & \\
\hline 20 & $(*)$ & & & & & .65 & \\
\hline 21 & $(*)$ & & & & & & .64 \\
\hline 22 & $(*)$ & & & & & & .64 \\
\hline 23 & $(*)$ & & & & & & .57 \\
\hline 24 & $(*)$ & & & & & & .60 \\
\hline \multicolumn{2}{|c|}{ Varianza por factor } & 8.01 & 10.96 & 6.90 & 9.74 & 6.26 & 13.11 \\
\hline \multicolumn{2}{|c|}{ Varianza Total Explicada } & 54.98 & & & & & \\
\hline
\end{tabular}

Nota: (*) Se ha omitido el contenido del item para resguardar los derechos de autor, según lo sugerido por los creadores del instrumento original. 


\section{Tabla 3.}

Matriz de correlaciones entre los factores de la escala.

\begin{tabular}{|c|c|c|c|c|c|c|}
\hline & 1 & 2 & 3 & 4 & 5 & 6 \\
\hline $\begin{array}{l}1 \text { Satisfacción } \\
\text { Autonomía }\end{array}$ & & $.461 * *$ & $.530 * *$ & $-.425^{* *}$ & $-.414 * *$ & $-.417 * *$ \\
\hline $\begin{array}{l}2 \text { Satisfacción } \\
\text { Relación }\end{array}$ & $.461 * *$ & & $.460^{* *}$ & $-.285^{* *}$ & $-.613 * *$ & $-.307 * *$ \\
\hline $\begin{array}{l}3 \text { Satisfacción } \\
\text { Competencia }\end{array}$ & $.530^{* *}$ & $.460^{* *}$ & & $-.379^{* *}$ & $-.382 * *$ & $-.532 * *$ \\
\hline $\begin{array}{l}4 \text { Frustración } \\
\text { Autonomía }\end{array}$ & $-.425 * *$ & $-.285^{* *}$ & $-.379 * *$ & & $.411^{* *}$ & $.508^{* *}$ \\
\hline $\begin{array}{l}5 \text { Frustración } \\
\text { Relación }\end{array}$ & $-.414 * *$ & $-.613 * *$ & $-.382 * *$ & $.411 * *$ & & $.460^{* *}$ \\
\hline $\begin{array}{l}6 \text { Frustración } \\
\text { Competencia }\end{array}$ & $-.417 * *$ & $-.307 * *$ & $-.532 * *$ & $.508^{* *}$ & $.460^{* *}$ & \\
\hline
\end{tabular}

Nota. ${ }^{* *}$ La correlación es significativa con $p<.01$

\section{Análisis Factorial Confirmatorio.}

El análisis factorial confirmatorio inicial de la escala de satisfacción y frustración de las necesidades psicológicas básicas mostró un adecuado ajuste de los datos al modelo, con un ratio $\chi^{2} / g l=1.75$; un CFI $=0.92$; un IFI $=0.92$; un TLI $=0.90$; un RMSEA de .05 (IC: .042 - .058) y un SRMR de .05. Los resultados anteriores apoyan la validez del modelo propuesto de seis factores a los datos utilizados.

Respecto a la consistencia interna de los factores, considerando el alfa ordinal que se presentan en la tabla 4 , los resultados muestran para el caso de la dimensión satisfacción de las necesidades psicológicas un alfa $=$ 0.90 y para la dimensión frustración de las necesidades un alfa $=0.86$. Los 
valores obtenidos para el caso de las seis subescalas o factores fue de un alfa $=0.71$ (satisfacción autonomía), un alfa $=0.73$ (satisfacción relación) y un alfa $=0.79$ (satisfacción competencia), mientras que se obtuvo un alfa $=.65$ (frustración autonomía), un alfa $=0.69$ (frustración relación) y finalmente un alfa $=0.70$ (frustración competencia), datos que se asemejan a los encontrados en el proceso de validación cruzada de la escala original en cuatro países, específicamente si se observan la consistencia interna encontrada en estudiantes universitarios en Perú (Chen et al., 2015).

\section{Tabla 4.}

Carga factorial y alfa ordinal por subescala y dimensión.

\begin{tabular}{lcc}
\hline \multicolumn{1}{c}{ Ítem } & Carga factorial & Alfa \\
\hline Satisfacción Autonomía & & $\mathbf{. 7 1}$ \\
Siento que tengo la libertad y la posibilidad & .61 & \\
de elegir las cosas que asumo. & & \\
$\left(^{*}\right)$ & .67 & \\
$\left(^{*}\right)$ & .75 & \\
$\left(^{*}\right)$ & .46 &
\end{tabular}

\section{Frustración Autonomía}

\section{Satisfacción Relación} me importan.

\section{Frustración Relación}

13 Me siento excluido del grupo al que quiero pertenecer.

$14 \quad(*)$

$15 \quad(*)$

$16 \quad(*)$ 
Satisfacción Competencia

17 Siento que puedo hacer las cosas bien.

$(*)$

$18 \quad(*)$

$19 \quad(*)$

20

Frustración Competencia

21 Tengo serias dudas acerca de si puedo hacer las cosas bien.

$22 \quad(*)$

$23 \quad(*)$

$24 \quad(*)$

Satisfacción de necesidades psicológicas Frustración de necesidades psicológicas

Nota: (*) Se ha omitido el contenido del item para resguardar los derechos de autor, según lo sugerido por los creadores del instrumento original.

\section{Discusión}

En los últimos años se ha reconocido en el marco de la TAD y en el ámbito académico que distintos tipos de variables personales, sociales, institucionales y contextuales pueden promover u obstaculizar la motivación intrínseca de los estudiantes, y por lo tanto satisfacer o frustrar las necesidades psicológicas básicas de autonomía, competencia y relación, con directas implicancias en el autoconcepto, autorregulación del aprendizaje, regulación motivacional, satisfacción académica y en el desempeño académico (Jeno \& Diseth, 2014; Méndez-Giménez, Fernández-Rio \& Cecchini, 2013; Moreno, Silveira \& Alias, 2015; Ümmet, 2015).

El propósito del estudio fue analizar las propiedades psicométricas, mediante un análisis factorial confirmatorio, de la Escala de Satisfacción y Frustración de las Necesidades Psicológicas Básicas (ESFNPB) identificadas por la teoría de la autodetermianción (Deci \& Ryan, 2000b), para el contexto de la educación superior en una muestra de estudiantes universitarios de distintas facultades y carreras pertenecientes a una universidad Chilena. 
El análisis factorial confirmatorio realizado permite apoyar la estructura de seis factores de la escala, lo que coincide con los resultados obtenidos en la propuesta original del instrumento realizada con estudiantes universitarios con edades comprendidas entre 16 y 32 años en cuatro países diferentes y la reciente validación de la escala realizada con estudiantes universitarios de distintas instituciones de educación superior de Portugal con edades compendiadas entre 18 y 37 años de edad (Chen et al., 2015; Cordeiro et al., 2016).

La validación de la ESFNPB realizada con estudiantes universitarios chilenos obtuvo aceptables valores de consistencia interna, un alfa ordinal $=.90$ y .86 para la dimensión satisfacción de las necesidades y frustración de las necesidades respectivamente. Para el caso de las seis subescalas los valores del alfa ordinal oscilaron entre .65 y .79, datos que se asemejan a los encontrados en el proceso de validación cruzada de la escala original, en particular si se observan la consistencia interna encontrada en estudiantes universitarios en Perú (Chen et al., 2015), resultados que también apoyan la distinción que realiza la escala para medir separadamente satisfacción y frustración de la necesidades psicológicas. Por su parte los 24 ítems que componen la escala presentaron adecuadas propiedades psicométricas, siendo significativos todos los valores de las saturaciones factoriales estandarizadas, así mismo el total de los ítems explican el 55\% de la varianza total. Además los resultados demostraron la existencia de una correlación negativa y significativa entre los factores que miden satisfacción y frustración de las necesidades psicológicas, corroborando la estructura de seis factores propuesta por Chen et al. (2015).

Según lo expuesto los resultados iniciales obtenidos mostraron un ajuste adecuado del modelo a los datos apoyando la validez del modelo propuesto de seis factores que miden satisfacción y frustración de las necesidades psicológicas básicas de autonomía, competencia y relación en el ámbito educativo, apoyando preliminarmente su uso en estudiantes universitarios 
en el contexto de la educación superior chilena (Moreno, Silveira \& Alias, 2015).

Al respecto y en el marco de $\mathrm{TAD}$, se reconoce que la motivación tiene una naturaleza multidimensional con distintos niveles situados en un continuo, desde el nivel más alto de autodeterminación y por lo tanto con elevada motivación intrínseca, hasta el nivel más bajo denominada amotivación (Moreno, Silveira \& Alias), siendo influenciadas por la satisfacción o frustración de las necesidades psicológicas básicas, desempeñando un papel fundamental en la salud psicológica de los estudiantes, en el desempeño académico y en la decisión de permanecer o abandonar los estudios universitarios.

En el ámbito de la educación superior distintas variables personales, sociales, institucionales y contextuales pueden apoyar o frustrar la satisfacción de las necesidades psicológicas básicas, por lo que resulta importante a nivel práctico, contar con un instrumento con adecuadas propiedades psicométricas que mida estas necesidades con el objetivo de identificar distintos ámbitos de intervención, tanto a nivel institucional como personal, para mejorar el proceso de integración académica y la permanencia en el contexto universitario.

Las limitaciones de la investigación se relacionan por una parte con el tipo de muestra utilizada, si bien participaron estudiantes adscritos a distintas carreras y facultades todos pertenecen a una misma institución universitaria por lo que se puede considerar que es una muestra bastante homogénea, futuras investigaciones para apoyar la validez y confiabilidad de la escala debiesen ampliar la muestra con otros estudiantes e instituciones para disminuir probables sesgos. Así también otra de las limitaciones del estudio es su carácter transversal y la falta de apoyo que evidencien validez convergente y/o divergente con otras escalas.

No obstante, los resultados obtenidos con el estudio aportan antecedentes preliminares para la utilización de la ESFNPB con estudiantes universitarios 
chilenos, es un aporte en el campo de la investigación en educación terciaria en relación al tema de la necesidades psicológicas y sirve de base para que futuras investigaciones realicen otros análisis para explorar la estructura factorial propuesta y la relación que puede existir con otras variables que inciden en la permanencia o abandono en la educación superior.

\section{Agradecimiento:}

Este trabajo se realizó con el apoyo del proyecto FONDECYT N 1161502 "Modelo explicativo de la permanencia y el abandono de los estudios universitarios, basado en procesos cognitivo motivacionales".

\section{Referencias}

Abello, R., Díaz, A., Pérez, M. V., Almeida, L. S., Lagos, I., González, J., \& Strickland, B. (2012). Vivencias e implicación académica en estudiantes universitarios: adaptación y validación de escalas para su evaluación. Estudios pedagógicos, 38(2), 7-19. Doi: http://dx.doi.org/10.4067/ S0718-07052012000200001

Agar, L. (2004). La ética de la investigación en ciencias sociales en el contexto de la globalización: de la investigación cuantitativa a la cualitativa. Acta bioethica, 10(1), 65-68. Doi: http://dx.doi.org/10.4067/ S1726-569X2004000100008

Ato, M., López, J., \& Benavente, A. (2013). Un sistema de clasificación de los diseños de investigación en psicología. Anales de Psicología, 29(3), 1038-1059. Doi: http://dx.doi.org/10.6018/analesps.29.3.178511

Barahona, P. (2014). Factores determinantes del rendimiento académico de los estudiantes de la Universidad de Atacama. Estudios pedagógicos, 40(1), 25-39. DOI: http://dx.doi.org/10.4067/S0718-07052014000100002

Boomsma, A. (2000). Reporting analyses of covariance structures. Structural Equation Modelling: A Multidisciplinary Journal, 7(3), 461-483. Doi: http://dx.doi.org/10.1207/S15328007SEM0703_6 
Byrne, B. (2009). Structural Equation Modeling with AMOS: Basic Concepts, Applications, and Programming. Mahwah, NJ: Lawrence Erlbaum Associates. Doi: https://doi.org/10.4324/9780203805534

Carranza, R., \& Apaza, E. (2015). Autoconcepto académico y motivación académica en jóvenes talento de una universidad privada de Tarapoto. Propósitos y Representaciones, 3(1), 233-263. Doi: http://dx.doi. org/10.20511/pyr2015.v3n1.72

Catalán, X., \& Santelices, M. V. (2014). Rendimiento académico de estudiantes de distinto nivel socioeconómico en universidades: el caso de la Pontificia Universidad Católica de Chile. Calidad en la educación, (40), 21-52. Doi: http://dx.doi.org/10.4067/S0718-45652014000100002

Cordeiro, P., Paixão, P., Lens, W., Lacante, M. \& Luyckx, K. (2016). The Portuguese Validation of the Basic Psychological Need Satisfaction and Frustration Scale: Concurrent and Longitudinal Relations to Well-being and Ill-being. Psychologica Belgica, 56(3), 193-209. Doi: http://doi. org/10.5334/pb.252

Curran, P. J., West, S. G. \& Finch, J. F. (1996). The Robustness of Test Statistics to Nonnormality and Specification Error in Confirmatory Factor Analysis. Psychological Methods, 1(1), 16-29. Doi: http:// psycnet.apa.org/doi/10.1037/1082-989X.1.1.16

Chen, B., Vansteenkiste, M., Beyers, W., Boone, L., Deci, E. L., Van der Kaap-Deeder, J., Duriez, B., Lens, W., Matos, L., Mouratidis, A., Ryan, R. M., Sheldon, K. M., Soenens, B., Petegem, S. V. \& Verstuyf, J. (2015). Basic psychological need satisfaction, need frustration, and need strength across four cultures. Motivation and Emotion, 39(2), 216-236. Doi: https://doi.org/10.1007/s11031-014-9450-1

Chilca, L. (2017). Autoestima, hábitos de estudio y rendimiento académico en estudiantes universitarios. Propósitos y Representaciones, 5(1), 71-127. Doi: http://dx.doi.org/10.20511/pyr2017.v5n1.145

Deci, E. L. \& Ryan, R. M. (2000a). Questionnaires: Basic Psychological Needs Scales. Recuperado de: http://www.psych.rochester.edu/SDT/ measures/needs.html 
Deci, E. L., \& Ryan, R. M. (2000b). The "What" and "Why" of Goal Pursuits: Human Needs and the Self-Determination of Behavior. Psychological Inquiry, 11, 227-268. http://dx.doi.org/10.1207/S15327965PLI1104_01

Doménech, F. B., \& Gómez, A. A. (2011). Relación entre las necesidades psicológicas del estudiante, los enfoques de aprendizaje, las estrategias de evitación y el rendimiento. Electronic Journal of Research in Educational Psychology, 9(24), 463-496. Recuperado de: http://www. investigacion- psicopedagogica.org/revista/new/ContadorArticulo. php?597

Donoso, S., \& E. Schiefelbein, (2007) Análisis de los modelos explicativos de retención de estudiantes en la universidad: una visión desde la desigualdad social. Estudios pedagógicos, 33(1), pp. 7, 27. https://doi. org/10.4067/s0718-07052007000100001

Elosua, P \& Zumbo, B. (2008). Coeficientes de fiabilidad para escalas de respuesta categórica ordenada. Psicothema, 20(4), 896-901. Recuperado de: $h$ ttp://www.psicothema.com/psicothema.asp?id=3572

Faye, C., \& Sharpe, D. (2008). Academic Motivation in University: The Role of Basic Psychological Needs and Identity Formation. Canadian Journal of Behavioural Science/Revue canadienne des sciences du comportement, 40(4), 189. Doi: http://psycnet.apa.org/doi/10.1037/ a0012858

Fernández-Hileman, M. R., Corengia, Á. \& Durand, D. (2014). Deserción y retención universitaria: una discusión bibliográfica. Pensando Psicología, 10(17), 85-96. Doi: https://doi.org/10.16925/pe.v10i17.787

Garbanzo, M. V. (2012). Factores asociados al rendimiento académico en estudiantes universitarios, una reflexión desde la calidad de la educación superior pública. Revista Educación, 31(1), 43-63. Recuperado de: http://www.redalyc.org/articulo.oa?id=44031103

Garcés, C. R., \& Arriagada, C. G. (2015). Capacidad predictiva de las notas en enseñanza media sobre el rendimiento en pruebas de selección universitaria: el caso chileno. Aula Abierta, 43(2), 61-68. Doi: https:// doi.org/10.1016/j.aula.2015.03.002 
Hair, J. F., Black, W. C., Babin, B., Anderson, R. E. \& Tatham, R. L. (2006). Multivariate Data Analysis (6 ${ }^{\mathrm{a}}$ ed.). Upper Saddle River, NJ: PrenticeHall.

Hu, L., \& Bentler, P. M. (1999). Cutoff Criteria for Fit Indexes in Covariance Structure Analysis: Conventional Criteria Versus New Alternatives. Structural Equation Modeling, 6, 1-55. Doi: http://dx.doi. org/10.1080/10705519909540118

Jeno, L. M., \& Diseth, Å. (2014). A Self-determination Theory Perspective on Autonomy Support, Autonomous Self-regulation, and Perceived School Performance. Reflecting Education, 9(1), 1-20. Recuperado de: http:// www.reflectingeducation.net/index.php/reflecting/article/view/121/125

Kline, R. B. (2005). Principles and Practice of Structural Equation Modeling (2.a. ed.). New York, NY: The Guilford Press. Doi: https://doi. org/10.1177/1049731509336986

León, J., Domínguez, E., Núñez, J. L., Pérez, A., \& Martín-Albo, J. (2011). Traducción y validación de la versión española de la Échelle de Satisfacción des Besoins Psychologiques en el contexto educativo. Anales de psicología, 27(2), 405-411. Recuperado de: http://www. redalyc.org/articulo.oa? $\mathrm{id}=16720051017$

Méndez-Giménez, A., Fernández-Río, J., \& Cecchini Estrada, J. A. (2013). Papel importante del alumnado, necesidades psicológicas básicas, regulaciones motivacionales y autoconcepto físico en educación física. Cuadernos de Psicología del Deporte, 13(1), 71-82. Doi: https://doi. org/10.4321/s1578-84232013000100008

Moreno J., Silveira Y., \& Alias, A. (2015). Modelo predictivo para la mejora de la percepción de competencia y rendimiento académico en estudiantes universitarios. REDU: Revista de Docencia Universitaria, 13(2), 9. Doi: https://doi.org/10.4995/redu.2015.5443

Miñano, P., \& Castejón, J. L. (2011). Variables cognitivas y motivacionales en el rendimiento académico en Lengua y Matemáticas: un modelo estructural. Journal of Psychodidactics, 16(2). Doi: https://doi. org/10.5944/educxx1.19052 
Mondragón, L. (2007). Ética de la investigación psicosocial. Salud Mental, 30(6), 25. Recuperado de: http://www.redalyc.org/pdf/582/58230604. pdf

Ryan, R. M., \& Deci, E. L. (2000a). Intrinsic and extrinsic motivations: Classic definitions and new directions. Contemporary educational psychology, 25(1), 54-67. Doi: http://dx.doi.org/10.1006/ceps.1999.1020

Ryan, R. M., \& Deci, E. L. (2000b). La Teoría de la Autodeterminación y la Facilitación de la Motivación Intrínseca, el Desarrollo Social, y el Bienestar. American Psychologist, 55(1), 68-78. Doi: http:// doi.10.1037110003-066X.55.1.68

Roth, E. (2012). Análisis multivariado en la investigación psicológica: Modelado predictivo y causal con SPSS y AMOS. La Paz, Bolivia: UCB/SOIPA.

Shumacker, R., \& Lomax, R. (1996). A Beginner's Guide to Structural Equation Modeling. Mahwah, USA: Erlbaum.

Stover, J. B., Uriel, F., De la Iglesia, G., Hoffman, A. F., \& Liporace, M. F. (2014). Rendimiento académico, estrategias de aprendizaje y motivación en alumnos de Escuela Media de Buenos Aires. Perspectivas en Psicología, 11(2), 10-20. Recuperado de: http://www.redalyc.org/ articulo.oa? $\mathrm{id}=483547666002$

Tian, L., Chen, H., \& Huebner, E. S. (2014). The Longitudinal Relationships Between Basic Psychological Needs Satisfaction at School and Schoolrelated Subjective Well-being in Adolescents. Social Indicators Research, 119(1), 353-372. Doi: https://doi.org/10.1007/s11205-013-0495-4

Ümmet, D. (2015). Self Esteem among College Students: A Study of Satisfaction of Basic Psychological Needs and Some Variables. Procedia-Social and Behavioral Sciences, 174, 1623-1629. Doi: https:// doi.org/10.1016/j.sbspro.2015.01.813

Valenzuela, J., Muñoz, C., Silva-Peña, I., Gómez, V., \& Precht, A. (2015). Motivación escolar: Claves para la formación motivacional de futuros docentes. Estudios pedagógicos, 41(1), 351-361. Doi: https://doi. org/10.4067/s0718-07052015000100021

Vlachopoulos, S. P. y Michailidou, S. (2006). Development and Initial Validation of a Measure of Autonomy, Competence, and Relatedness in 
Exercise: The Basic Psychological Needs in Exercise Scale. Measurement in Physical Education and Exercise Science, 103, 179-201. Doi: http:// dx.doi.org/10.1207/s15327841mpee1003_4

Wilson, P. M., Rogers, W. T., Rodgers, W. M. y Wild, C. (2006). The Psychological Need Satisfaction Excercise Scale. Journal of Sport \& Excercise Psychology, 28(3), 231-251. Doi: https://doi.org/10.1123/ jsep.28.3.231 